\title{
Esse sequitur operari, o el nuevo giro de la teoría sociológica contemporánea: Bourdieu, Archer, Luhmann
}

\author{
Esse sequitur operari, Or the New Turn of Contemporary Sociological Theory: Bourdieu, \\ Archer, Lubmann
}

Aldo Mascareño
Centro de Investigación Núcleo Milenio Modelos de Crisis, Universidad Adolfo Ibáñez, Chile

\section{RESUMEN}

El artículo sostiene que en la teoría sociológica contemporánea es posible reconocer un conjunto de teorías operativas para las cuales el mecanismo generativo de lo social reside en el centro de su arquitectura teórica. Para estas teorías el concepto de operación juega un rol central en la descripción de la emergencia de la sociedad, mientras que el concepto de estructura funciona como un dispositivo limitacional y reflexivo que informa a la operación acerca de las posibilidades contingentes de la dinámica social. El artículo argumenta que la lógica de la práctica de Pierre Bourdieu, la dislocación temporal entre agencia y estructura, así como el rol intermediario de la interacción en Margaret Archer, y el concepto de comunicación de Niklas Luhmann como un evento de selección tripartito, representan opciones alternativas en la explicación operativa de la emergencia de lo social. Las conclusiones examinan los rasgos de las teorías operativas en la perspectiva de extraer las consecuencias para conceptos básicos de las ciencias sociales tales como tiempo, espacio, la distinción micro/macro y la posición de las normas en la teorización sociológica.

Palabras Clave: Operación; Estructura; Práctica; Morfogénesis; Sistema social; Teoría sociológica

\section{ABSTRACT}

The article argues that in contemporary sociological theory it is possible to recognize a set of operational theories for which the generative mechanism of the social lies at the center of their theoretical architecture. For these theories, the concept of operation plays a pivotal role in describing the emergence of society, while the concept of structure functions as a limitative, reflexive device that informs the operation about the contingent possibilities of social dynamics. The article contends that Pierre Bourdieu's logic of practice, Margaret Archer's temporal dislocation between agency and structure as well as the intermediary role of interaction, and Niklas Luhmann's concept of communication as a threefold selection event represent alternative options in operatively explaining social emergence. Conclusions examine the features of operational theories with a view to drawing the theoretical consequences for basic concepts of social sciences such as time, space, the distinction micro/macro, and the position of norms in sociological theorizing.

KEYWORDS: Operation; Structure; Practice; Morphogenesis; Social system; Sociological theory

MAD, N ${ }^{\circ} 37$ (2017), PP. 54-74

DOI: $10.5354 / 0718-0527.2017 .47272$

(C) CC BY-NC 3.0 CL 


\section{INTRODUCCIÓN ${ }^{1}$}

No hay duda que la tradición dominante de la metafísica occidental ha estado profundamente marcada por la primacía aristotélica de la substancia, por la ontología de 'las cosas'. Paralela a esta, sin embargo, la denominada filosofía de procesos reclama su lugar. En ella, el axioma operari sequitur esse (la operación deriva del ser) se invierte en la fórmula esse sequitur operari (el ser deriva de la operación). A diferencia situar el centro analítico en el objeto, lo que aquí se enfatiza es el proceso, el evento, el acontecimiento, la contingencia. Una genealogía de esta tradición debiera contar con Heráclito, Gottfried Leibniz, Charles S. Peirce, Alfred N. Whitehead y su escuela (Rescher 1996). Y seguramente debiera también incluir la crítica de la metafísica de la presencia de Jacques Derrida (1997), la denominada filosofía post-fundacional (Marttila 2015), algunos componentes del giro reflexivo en antropología y ciencias sociales (Alladi 2013; Bourdieu \& Wacquant 1992) e importantes elementos en las teorías sociológicas de Pierre Bourdieu, Margaret Archer y Niklas Luhmann, especialmente si es posible demostrar que las arquitecturas de estas teorías presentan una opción por la operación antes que por las estructuras en sus propuestas acerca de la construcción de lo social.

Como es claro, al centrarme en estos tres autores de la sociología contemporánea no pretendo en este artículo ofrecer un panorama metateórico exhaustivo de la tradición sociológica leída desde una distinción entre operación y estructura o desde el punto de vista de lo que llamaré siguiendo una formulación de Armin Nassehi (2006)- "teoría operativa". Sin embargo, una reconstrucción teórica de esta naturaleza parece plausible si uno atiende a momentos clave de esa tradición o de sus fuentes. Probablemente el primer aviso de la importancia de la operación en la construcción de lo social vino desde Karl Marx (1978: 7) en la Tesis 8: "Toda vida social es esencialmente práctica. Todos los misterios que inducen a la teoría hacia el misticismo encuentran su solución racional en la praxis humana y en la comprensión de esa praxis". El segundo aviso podría encontrarse en el giro lingüístico: el mundo no es un factum, su construcción se opera el lenguaje, o en palabras de Ludwig Wittgenstein (1986: 11): “el término juego de lenguaje busca poner de relieve el hecho de que hablar el lenguaje es

\footnotetext{
${ }^{1}$ El presente trabajo se realizó en el marco del Núcleo Milenio Modelos de Crisis (NS 130017)
} 
parte de una actividad, o de una forma de vida". Y el tercero está en las discusiones sobre autorreferencia en la cibernética, las ciencias cognitivas y, particularmente, en él énfasis en el mecanismo generativo de la metabiología de Humberto Maturana (2009: 75): "La reformulación de la experiencia (fenómeno) $[\ldots]$ ha de explicarse en la forma de un mecanismo generativo que, si realizado por un observador tipo en su dominio de experiencias, le permitiría, como consecuencia de su operación, tener en su dominio de experiencia la experiencia que ha de ser explicada". Bajo un criterio operativo, la distinción weberiana entre acción social (con sentido) y acción no social (Weber 1992) quedaría fuera, pues uno debe presuponer la anterioridad del sentido para distinguir entre acción con sentido y acción no social. En ese caso, se mantiene la pregunta: ¿cuál es la operación que produce sentido? Asimismo, los tres momentos del desarrollo teórico de Talcott Parsons mostrarían un problema similar: el unity act presupone valores, medios y fines (Parsons 1968), el sistema social presupone el sistema cultural (Parsons 1991), y el AGIL atribuye una estructura funcional a la sociedad (Parsons et al. 1970) cuya génesis no queda resuelta. ${ }^{2}$ Dicho en términos de una fórmula, en un mundo postestructural, la pregunta debe ser originalmente operativa: no 'qué', sino 'cómo'.

Me parece que desde finales del siglo XX asistimos en teoría sociológica a la consolidación de una tendencia que intenta dar con el mecanismo operativo que genera lo social. 'Cómo' emerge lo social -y no 'qué' es lo social- es la pregunta central. Si este es un nuevo giro, el 'giro operativo', que agregar al desarrollo de la teoría sociológica (además del giro lingüístico, el jurídico y el reflexivo) es aún una pregunta abierta. Si bien lo propongo como posibilidad, creo que es factible identificar ya alguna unidad general. Las construcciones teóricas operativas no arrancan desde componentes estructurales, desde principios naturales o de la facticidad de los datos. Cuando la pregunta es 'cómo', la práctica teórica se convierte en la unidad de la diferencia entre teoría y praxis que reproduce permanentemente esa diferencia por medio de una operación cuya función es mostrar, en la propia descripción, cómo emerge el fenómeno social que se quiere explicar. Las teorías operativas evitan presuponer lo social antes de que lo social emerja y para ello proponen un mecanismo generativo que dé cuenta de esa emergencia (Mascareño 2008).

${ }^{2}$ En el caso de Parsons, no obstante, siempre habría que evaluar cómo la resolución del problema de la doble contingencia opera en sentido contrario a esta visión estructural. 
Mi hipótesis es que en la oferta sociológica contemporánea hay tres de estas teorías generales que son, en propiedad, teorías operativas: la teoría del sentido práctico de Pierre Bourdieu, la teoría morfogenética de Margaret Archer y la teoría de sistemas de Niklas Luhmann. ${ }^{3}$ Sostengo también que en las tres pueden aparecer ciertos riesgos estructuralistas con menor o mayor intensidad. Mientras en Bourdieu el habitus parece sobrestructurar la lucha de capitales en los campos y en Archer la estratificación ontológica en el nivel de la agencia establece demasiados presupuestos a la operación, en Luhmann la atribución de estabilidad a la autopoiesis sistémica puede limitar la dinámica de la operación. No busco interpretar estos riesgos como resabios de una metafísica de la presencia en la teoría sociológica que habría que eliminar, para lograr algo así como una teoría procesualista u operativa pura de lo social. Ningún científico social podría dudar ni hoy ni antes de la alta capacidad de las estructuras sociales para resistir la práctica de los agentes, para establecer rangos de variabilidad a la operación, e incluso para retornar a momentos prerreforma o prerrevolución luego de importantes transformaciones morfogenéticas. El problema no es, si operación o si estructura. La fórmula esse sequitur operari no indica negación de las objetualidades, sino su postposición en relación a la operación en la comprensión de la emergencia de lo social. Dicho en términos de la codificación luhmanniana, mi intención es mostrar que la operación es el valor de conexión en las teorías indicadas, es decir, el que genera las operaciones teóricas que, a su vez, reconstruyen un correlato de la génesis de lo social; mientras que la estructura es el valor reflexivo, esto es, el que le muestra a la operación márgenes, en todo caso móviles, de "limitacionalidad": "La limitacionalidad viene dada en un determinado ámbito de enunciados cuando las negaciones no operan en vacío, sino que con el enunciado de que algo no es A se contribuye a la determinación de A" (Luhmann 2013: 64).

Para desplegar esta hipótesis comienzo identificando la lógica de la práctica en Bourdieu como fundamento operativo de su teoría y discuto posibilidades de riesgo estructuralista en ella (1). Continuo con la diferencia de temporalidad entre agencia y estructura de Archer como operación fundamental del enfoque morfogenético e identifico los elementos ontológicos de

\footnotetext{
${ }^{3}$ Sin duda este ejercicio también puede aplicarse a otras teorías contemporáneas a las que, en principio, parece plausible señalar como teorías operativas: la operación de control en la teoría de red de Harrison C. White (2008), la operación de relación en la denominada sociología relacional (Emirbayer 1997; Donati 2011), la operación de conexión en teorías socio-cognitivas (Varela et al. 1993). Por efectos de espacio, dejo a estas teorías fuera del análisis de este artículo.
} 
la agencia que obstruyen el libre movimiento de la operación (2). Sigo luego con la formulación radical de la doble contingencia en Luhmann y observo el riesgo de atribuir estabilidad a la autopoiesis de sistemas sociales para la dinámica de la operación (3). Finalmente, extraigo de lo anterior algunas conclusiones en relación a los rendimientos de teorías operativas.

\section{Operación COMO JUEGO SOCIAL: PIERRE BOURDIEU}

Si a nivel de comparaciones teóricas, lo que une a Bourdieu, Archer y Luhmann es que son teorías operativas en el sentido descrito, es decir, teorías que muestran, en la propia descripción, cómo emerge el fenómeno social que quieren explicar, tiene que haber para ellas un problema de referencia común, también a nivel teórico, que quieran resolver. En mi opinión, dicho problema es que ninguna de ellas se siente realmente satisfecha con atribuir a los agentes o a las estructuras la primacía en la explicación de lo social. Archer reflexiona esto con ayuda de los conceptos de individualismo y colectivismo; Luhmann lo hace por medio de la diferencia entre tradición humanista y estructural-funcionalismo; y Bourdieu distinguiendo entre subjetivismo y objetivismo.

Para Bourdieu, tanto el subjetivismo (cuya referencia es Jean Paul Sartre y la teoría de la elección racional) como el objetivismo (representado en Ferdinand de Seaussure y Claude Lévi-Strauss) limitan la comprensión de lo social, no solo porque no toman en cuenta lo que la otra posición puede aportar al problema, sino que la referencia a sus propias premisas los hace ciegos "a las condiciones de producción y funcionamiento del sentido del juego social que permite vivir como algo evidente el sentido objetivado en las instituciones" (Bourdieu 2007: 45s.). El problema, por tanto, no es solo teórico sino también epistemológico. Puesto que, para Bourdieu (1997), "lo real es relacional", al funcionar bajo un esquema de reductibilidad, tanto subjetivismo como objetivismo no tienen efectivo acceso a lo social. Una y otra opción son obstáculos para sí mismas: invitan a hacerse preguntas (como aquella por la constitución de lo social) que en realidad no pueden responder, porque la respuesta está en algo que no ven. La invitación de Bourdieu (2007: 85s.) es "retornar a la práctica, ámbito de la dialéctica del opus operatum y del modus operandi, de los productos objetivados y de los productos incorporados de la práctica histórica, de las estructuras y de los habitus". 
La fórmula para la superación de esta dicotomía es en Bourdieu la praxis o la lógica de la práctica. En palabras del autor: "Hay que reconocerle a la práctica una lógica que no es la de la lógica para evitar pedirle más lógica de la que puede dar y así condenarse ya sea a extraerle incoherencias, o bien a imponerle una coherencia forzada" (Bourdieu 2007: 137). La coherencia práctica es, por tanto, una coherencia no unitaria, en la que hay que contar con regularidades, pero también con incoherencias. Esto hace recordar la fórmula gödeliana de la incompletitud de los sistemas lógicomatemáticos que, aplicada a este caso, significa que la lógica de la práctica es completa solo cuando se reconoce su incompletitud. En la relacionalidad de lo social no hay un orden a priori, las mismas 'cosas' pueden recibir significaciones diferentes -incluso opuestas- dependiendo del universo de práctica del que se trate. Esto implica dos cosas relevantes para los fines de mi hipótesis inicial: a) que el sentido práctico es una operación y, como toda operación, es sustancialmente un evento presente, y b) que cuando a la práctica se la considera operación, se le restituye su sentido "al referirla a las condiciones reales de su génesis, es decir, a las condiciones en las cuales se hallan definidas las funciones que ella cumple y los medios que emplea para lograrlas" (Bourdieu 2007: 155). Porque es sustancialmente presente, la operación no tiene a priori, y porque es genética, la operación es origen de sentido.

En la teoría de los campos la operación se expresa como lucha de intereses, disputa, competencia. Ahí es donde el sentido del juego social se pone en práctica como relación de poder regulada por una distribución de capitales acumulados en luchas anteriores. En este caso, la distribución de capitales acumulados funciona como limitacionalidad de la operación, como su valor reflexivo: operación, pero en este espacio'. No es que los capitales se impongan sobre la operación, pues para acumularlos hubo también que operar. Esse sequitur operari. Tampoco es que los capitales determinen que ha de realizarse un tipo específico de operación, pues el escenario es de lucha, es decir, de operación práctica de la que puede surgir uno u otro resultado. Justamente ahí se encuentra el antecedente para la transformación social en términos de subversión o de conservación de la distribución de capitales (Bourdieu 2002). Morfogénesis o morfostasis en términos de Archer (2009). La propia práctica teórica es una lucha de este tipo: Bourdieu se encuentra en ella haciéndose espacio entre el subjetivismo y el objetivismo. "Autología" llama Luhmann (1984) a esta capacidad de autoposiciona- 
miento de la teoría al interior de la propia teoría. Una capacidad que distingue toda superteoría (Luhmann 2013).

De cualquier modo, no es que los individuos lleguen como tabula rasa al momento práctico: "Para que funcione un campo, es necesario que haya algo en juego y gente dispuesta a jugar, que esté: dotada de los habitus que implican el conocimiento y reconocimiento de las leyes inmanentes al juego, de lo que está en juego, etcétera" (Bourdieu 2002: 120). La teoría del habitus ha sido criticada porque ella contiene la idea de que lo que el actor hace deriva de una estructura que lo supera, una estructura "estructurada y estructurante", en la que este siempre está inmerso (Archer 2004, 2012; Aguilar 2014). Bourdieu habría querido escapar del estructuralismo y volvería a caer en él con la teoría del habitus.

Es cierto que la primera parte de su definición de habitus parece dar sustento a esta crítica y mostrar también riesgos estructuralistas en su concepción de la emergencia de lo social. Bourdieu define el habitus como "sistemas de disposiciones duraderas y transferibles, estructuras estructuradas dispuestas a funcionar como estructuras estructurantes" (Bourdieu 2007: 86). Sin embargo, lo que inmediatamente sigue introduce un giro decisivo para mi interpretación: "es decir [continúa Bourdieu], como principios generadores y organizadores de prácticas y de representaciones" (Bourdieu 2007: 86 -destacado mío). La crítica a Bourdieu ha tomado en cuenta principalmente la primera parte de la definición del habitus y ha subvalorado la importancia de la segunda. El concepto de habitus se dispone, en realidad, contra el supuesto de autotransparencia de la acción que subyace en la teoría de la acción racional. Como lo ha indicado Nassehi (2004: 168): "El concepto de habitus intenta mostrar que la acción es un acontecimiento cuyo sentido social rebasa con mucho las reflexiones significativas del actor". En la acción hay más sentido del que el actor atribuye. A mi entender, este concepto de habitus cumple estrictamente con el principio de limitacionalidad expuesto más arriba: la generación y organización de prácticas y representaciones (operación) no ocurre en el vacío sino en un medium de elementos y relaciones en marcha. Entendido de esta forma, el habitus no es solo una estructura, es también una operación que genera y organiza en el espacio de la lógica de la práctica. La pregunta que se abre es entonces: ¿operari sequitur esse o esse sequitur operari? ¿La operación sigue al ser o el ser a la operación? Bourdieu (2007: 90) rechaza explícitamente la primera opción: "No hay nada más engañoso que la ilusión retrospectiva que hace aparecer el conjunto de huellas de una vida, tales como las obras 
de un artista o los acontecimientos de una biografía, como la realización de una esencia que les era preexistente". La clave es más bien operativa: la dialéctica entre intención de objetivación e intención objetiva en la producción artística, la confrontación entre esquemas producidos en la práctica y la práctica que cambia o reproduce esos esquemas, o en palabras de Bourdieu (2007: 90): "la confrontación a la vez necesaria e imprevisible del habitus con el acontecimiento que no puede ejercer sobre el habitus una incitación pertinente a menos que este lo arranque a la contingencia del accidente y lo constituya en problema al aplicarle los principios mismos de su solución".

El juego social de la lógica de la práctica está entonces abierto a la praxis. Por cierto, tiene predilecciones derivadas de las operaciones pasadas, pero estas no deben entenderse como determinaciones sino con una función limitacional en el sentido descrito más arriba: El habitus "consiste de un conjunto de relaciones históricas (...) es creativo, inventivo, pero dentro de los límites de sus estructuras" (Wacquant 1992: 16, 19). Junto con el campo, constituyen un "espacio de juego que existe como tal solo en la medida que los jugadores entran en él" (Wacquant 1992: 19). La operación es finalmente la que instaura el juego social de la praxis. De este modo, tampoco habría que entender la praxis como una cuestión solo de seres humanos con voluntad, autoría, racionalidad y presencia. Un rendimiento de la teoría de Bourdieu es que un concepto cartesiano de sujeto como origen de sentido queda descentrado en la tensión y complementariedad que tiene lugar en la práctica entre habitus y las luchas del campo. En ese marco, el actor es un elemento de la dinámica operativa, no el héroe ni el fundamento; es cuerpo en relación de habitus, instituciones y capitales. El actor se deconstruye en su propio cuerpo. De este modo, la "acción [...] aparece finalmente aquí como vivencia, lo que enfatiza la orientación de Bourdieu al problema de referencia de la emergencia de estructuras sociales desde la praxis" (Nassehi 2006: 257).

Cuando el problema de referencia de una teoría es la emergencia de estructuras sociales, entonces: esse sequitur operari, el ser sigue a la operación. No puede ser de otro modo. Solo por medio de la identificación del mecanismo operativo (o generativo) es posible reconstruir la dinámica de la emergencia de aquello que se puede concebir como estructura, y solo por medio de la operación aplicada a estructuras se puede entender a estas como formas metaestables, es decir, cambiantes en rangos móviles de variabilidad. 


\section{OPERACIÓN COMO JUEGO MUTUO: MARGARET ARCHER}

No debe extrañar que Archer también recurra a la metáfora del juego para abordar su problema de referencia. Para indicar este problema, Bourdieu empleaba la distinción subjetivismo/objetivismo. Archer reemplaza esta por la distinción entre individualismo y colectivismo, pero el problema es el mismo: el individualismo reduce las estructuras a la acción de agentes y el colectivismo disuelve la agencia en estructuras. Para Archer, tanto agencia como estructura son estratos ontológicamente distintos y, en tanto lo son, gozan de poderes causales diferenciados. De tal modo, "si los diferentes estratos poseen propiedades diferentes e, inter alia, se asume que la estructura y la agencia son estratos separados por esa misma razón, es crucial entonces examinar su juego mutuo" (Archer 2009: 46).

Cuando Archer habla del juego mutuo entre estructura y agencia ya confirma que su teoría de lo social es operativa: lo social emerge en la operación del juego mutuo. Mientras que el juego social de Bourdieu se hacía operativo en las prácticas sociales, Archer rechaza el concepto de práctica de Bourdieu, en tanto este fusiona la autonomía de estructura y agencia y hace que se pierdan las propiedades causales emergentes de una y otra. Esto es lo que Archer (2009) llama "el pecado del conflacionismo central". Distinto al conflacionismo ascendente, para el cual la estructura es epifenómeno de la acción, y distinto al descendente, para el que los actores son autómatas estructurales o culturales, el conflacionismo central reduce ambos niveles ontológicos (agencia y estructura) a la ontología de la práctica.

Si se trata de una teoría operativa, es decir, una que muestra en la propia descripción, cómo emerge el fenómeno social que se quiere explicar, el rechazo de la práctica exige a Archer encontrar un lugar alternativo para situar la operación al interior de su arquitectura teórica. Mi respuesta es que, en la teoría de Archer, la operación se encuentra en dos niveles interrelacionados. El primero de ellos es la diferencia temporal existente entre estructura y agencia, y el segundo es el rol de intermediación de la agencia en la morfogénesis estructural. Explico ambos a continuación.

La perspectiva de Archer se enmarca dentro del realismo crítico (Bhaskar 1989). Este atribuye una estratificación ontológica al mundo social, en tanto reconoce la autonomía de estructura y agencia. Autonomía debe entenderse aquí, en sentido emergentista, como la capacidad operativamente producida de ejercer poderes causales propios e irreductibles sobre otros niveles (Archer 2009). Esto que podría parecer un doble es- 
tructuralismo que reifica ambas dimensiones, deja de serlo cuando se introduce la variable temporal: las estructuras sociales anteceden a la acción de las personas. O en una fórmula más explícita: la mayoría de los actores están muertos. Una generación es distinta de la anterior y cada una de ellas actúa en un mundo social que viene prefigurado estructuralmente por las generaciones anteriores. Como lo señala Archer en relación con la estructura del matrimonio: "actores previos a través de sus prácticas sociales previas constituían ellos mismos la institución del matrimonio con anterioridad en la historia (esto se refiere a agentes muertos hace tiempo)" (Archer 2009: 108). Por ello, el tiempo de la estructura tiene un horizonte más largo que el tiempo de la agencia. El juego mutuo de agencia y estructura es posible por este diferencial de temporalidad. Se trata de un juego mutuo, porque la agencia actúa, opera con sus poderes causales, no es 'mera portadora' de la presión estructural. Y puede actuar en dos sentidos: morfoestáticamente, es decir, reproduciendo la estructura existente, o morfogenéticamente, es decir, transformándola.

A la operación de este juego mutuo, Archer (2009) lo llama ciclo morfogenético/morfoestático. Analíticamente, en $t_{1}$ (momento temporal 1) se encuentra la estructura que viene de un ciclo anterior; entre $t_{2} \mathrm{y} \mathrm{t}_{3}$ se produce la interacción -en base a diversos proyectos y estrategias personalesque reproduce o modifica la estructura, y en $t_{4}$ se obtiene la estructura reproducida o modificada por la interacción. La agencia tiene, por tanto, un rol intermediador entre la estructura en $t_{1}$ y la estructura en $t_{4}$. La sociedad emerge de esta operación que es posible por la diferencia temporal de estructura y agencia, y el rol de intermediación de la agencia a partir de proyectos personales. Incluso si el resultado de la intermediación agencial de estructuras es la morfostasis, es decir, la reproducción de las estructuras, no puede prescindirse de la operación de intermediación para explicarla: alguien (personas, agentes, actores) tuvo que oponerse al cambio o alguien (personas, agentes, actores) tuvo que reproducir las ventajas del presente para inmunizarlo frente a la contingencia del futuro. En ambos casos, esse sequitur operari.

Sustantivamente, las teorías de Archer y Bourdieu son radicalmente distintas y contrapuestas en variados aspectos y consecuencias. Sin embargo, ambas recurren a una operación para resolver el problema de la emergencia de lo social. De un modo similar a Bourdieu, también en Archer se puede constatar la función de limitacionalidad de las estructuras. La 
agencia no ejerce sus poderes causales en el vacío, sino que los pone en práctica frente a los poderes causales de una estructura formada y reformada en ciclos morfogenéticos previos. Pero, así como el habitus en Bourdieu puede hacer suponer un riesgo de sobredeterminación de prácticas cuando se enfatiza solo el componente estructural, también en Archer me parece que la estratificación de la agencia en agentes, actores y personas genera un riesgo para la explicación operativa de lo social. El riesgo es que el nivel ontológico de la interacción que media entre la estructura en $\mathrm{t}_{1} \mathrm{y} \mathrm{t}_{4}$ también tiene un ciclo morfogenético, en el cual la agencia media entre el nivel ontológico de la persona (nivel de humanidad), el del agente (personas en colectividades con las mismas oportunidades de vida) y el del actor (persona en relación a colectividades y roles organizacionales). A esto Archer (2009) le llama doble (agentes) y triple (actores) morfogénesis.

El problema, en realidad, no está en la doble o triple morfogénesis de la interacción. Después de todo, la interacción continúa como operador en ambos casos. ${ }^{4} \mathrm{El}$ problema está en que Archer sitúa lo que denomina el principio de humanidad en la persona, esto es, un "anclaje a priori para comprensibilidad temporal de agentes y actores" (Archer 2009: 372). Archer sintetiza este principio de humanidad como la continuidad de la conciencia. Puesto en estos términos, el criterio no sería problemático, pues la continuidad de la conciencia bien puede entenderse como la continuidad del flujo de conciencia que opera en el sentido husserliano (y luhmanniano) de la intencionalidad y que es autónomo en relación con la operación social. Archer, sin embargo, no sigue este camino. Por medio de referencias a Marcel Mauss, Archer afirma que "se debe siempre sostener una distinción fundamental entre el concepto del yo que evoluciona (que por cierto es social) y el sentido universal del yo (que no evoluciona)" (Archer 2009: 374-5). Esta es una figura estructural por excelencia que puede aceptar la contingencia y operatividad del mundo (concepto del yo que evoluciona), porque sitúa fuera del mundo lo que no quiere que cambie (el sentido universal del yo) y lo reviste de necesidad al no quedar sometido a la evolución. Se trata de un procedimiento similar al empleado en la teodicea de Leibniz, en el

\footnotetext{
${ }^{4}$ Un problema teórico distinto es cómo se introduce un nivel de emergencia dentro de otro. Pues si la emergencia genera condiciones de autonomía (poderes causales), la morfogénesis de la agencia (doble y triple) quedaría situada dentro de la morfogénesis de lo social (entre agencia y estructura), con lo que se produce el problema lógico de una autonomía dentro de otra. No hay muchas respuestas posibles para esto: o la morfogénesis de la agencia no genera realmente condiciones de autonomía, o la morfogénesis social (entre agencia y estructura) es un derivado de la morfogénesis de la agencia (Mascareño 2008).
} 
que se defiende la sabiduría suprema del creador ante la imperfección de su creación: el mundo contingente es el mejor de los mundos posibles creado por un agente perfecto fuera del mundo (Marquard 2003). A este nivel, el 'sentido universal del yo' queda fuera de la contingencia de la historia y la evolución, no es explicable por ellas. El problema no está en sustentar la autonomía del individuo frente a la sociedad. La autonomía (poderes causales) del nivel de la agencia ya lo hace. El problema está en que la autonomía del nivel agencial se apoye en una estructura última invariante de la persona y no se sostenga por sí misma de manera operativa. En la doble y triple morfogénesis de la interacción parece funcionar el principio inverso al que funciona a nivel de lo social: porque la persona 'es' de algún modo, entonces se opera como se opera.

En este caso, no se puede interpretar el sentido universal del yo como función limitacional de la operación, pues la estructura no ha sido formada por ninguna operación. De alguna manera 'está ahí'. En relación al cuerpo, Archer (2009: 381) hace ver más inequívocamente lo que esto implica: "las personas vivas tienen que tener cuerpos, los cuerpos de los seres humanos son cuerpos animales, cuyas propiedades constituyen la esencia real del homo sapiens". El problema, en este caso, no son los cuerpos, sino que sus propiedades constituyan esencias. Pues cuando se trata de esencias, la operación es posterior al ser. Para presentar (o defender) la autonomía de la persona no parece necesario llegar tan lejos.

En libros posteriores, Archer $(2004,2007,2012)$ realiza un extenso despliegue de la forma en que la persona constituye el nivel agencial y se relaciona con el nivel estructural. Los conceptos que se emplean (especialmente conversación interna, reflexividad, relación) ponen énfasis en la operación, por ejemplo, en la fuerza motivacional de las personas para implementar su modus vivendi a través de proyectos agenciales y no vuelven sobre las propiedades que 'constituyen la esencia real del homo sapiens'. Más aún, en una formulación actual, Archer señala: "El sentido [del sí mismo] es continuo, pero no hay esencia del self que detectar" (Archer 2015: 99). Este libro reciente (escrito junto a Pierpalo Donati) busca realizar una suerte de acoplamiento teórico entre el realismo crítico y la sociología relacional. Esto último, aleja los riesgos estructuralistas, pero también supone alejarse del sentido universal del yo como elemento último de la morfogénesis de la agencia. 


\section{OPERACIÓN COMO COMUNICACIÓN: NIKLAS LUHMANN}

En el caso de Luhmann, el problema teórico de referencia se formula con un ojo puesto en el estructural-funcionalismo y otro en la tradición humanista. En relación con el primero, rechaza el estructuralismo y, con el segundo, rechaza la teoría de la acción. Es decir, se mantienen los mismos términos del problema de Bourdieu (objetivismo/subjetivismo) y de Archer (colectivismo/individualismo). El rechazo del estructuralismo se realiza como crítica al estructuralismo funcional de Parsons, para quien el problema de la doble contingencia (la duplicación de sentido de alter y ego frente a una misma situación) se resolvía por un horizonte normativo que escapaba a la operación, o que al menos no se hacía visible. La norma solucionaba el problema ordenando la doble contingencia, pero dejaba sin resolver el proceso por el cual se había producido la norma. Como lo ha sostenido Hugo Cadenas (2012: 211) en relación a la evaluación luhmanniana de Parsons: "Las estructuras [...] han de ser entendidas dentro de un horizonte temporal en el cual éstas emergen como consecuencia de la operación selectiva de sistemas sociales que, fruto de la operación de equivalencia funcional, concatenan selecciones como eventos con expectativas de futuro". El rechazo del humanismo, en tanto, constituye la crítica de la reducción de lo social a la acción e interacción humana. La acción es demasiado esporádica para sostener lo social, está cargada de presupuestos que Bourdieu llamaría subjetivistas: volición, intencionalidad, racionalidad. Pero, para Luhmann $(1984,2007)$, el problema principal de la acción es que está inscrita en algo más fundamental que la incluye y la produce. Esto es, la comunicación.

La comunicación es operación por excelencia. Se trata, en todo caso, de un concepto de comunicación como síntesis de selecciones concatenadas de alter y de ego. Mientras alter selecciona una información y sucesivamente una forma de expresarla, ego selecciona una comprensión al observar la expresión de alter (Luhmann 1984). Comunicación no es, por tanto, una acción; es una constelación de selecciones. La comunicación se desplaza de evento en evento; no en un sentido lineal, sino reticular. Tampoco, por tanto, en una dirección o ritmo temporal unívoco, pues incluso el tiempo es un derivado de la operación de comunicación; solo en ella se pueden distinguir horizontes temporales adecuados a cada programa sistémico. Igualmente, la acción queda comprendida como forma de expresión de un contenido informativo al interior de la comunicación: alguien habla, gesticula, baila, calla o simplemente no está. Todo ello gatilla la selección de una 
comprensión en ego que no tiene por qué ser la que alter tenía en mente. Es muy improbable que lo sea en realidad. Incluso aquel que no está, y que ni siquiera se enteró, puede ser operativamente incorporado en la comunicación como tercero excluido: quien había reservado una vivienda posteriormente vendida a una mejor oferta, quien trabaja informal y esporádicamente, y es contabilizado en las cifras de empleo del gobierno, quien recibe más archivos que revisar porque no estuvo en la reunión, quien es parte de un triángulo amoroso sin llegar a enterarse.

La selección de una compresión por parte de ego tiene alta independencia de la selección de información y de la conducta expresiva de alter. No obstante, ambas quedan ligadas por la situación, por la referencia a un mismo ámbito de sentido que, sin embargo, se duplica para alter y para ego. Esto es doble contingencia. Mientras Parsons situó las normas por sobre esta constelación operativa, Luhmann (1998) radicaliza la operación y resuelve la doble contingencia por el enlace de una situación de comunicación con la siguiente, es decir, por el enlace de doble contingencia con más doble contingencia. Este enlace operativo es el mecanismo generativo de lo social. Es cierto que implica sentido, pero el sentido no es anterior, sino que se actualiza en la operación; no preexiste como estructura de control, es cooriginario con la comunicación. Esse sequitur operari.

La reiteración de una selección de sentido en comunicaciones sucesivas genera identidades y sistemas (Luhmann 2005a). ¿Por qué se producen estas recursiones que forman sistemas? Porque resuelven problemas que las mismas recursiones hacen aparecer como tales. Como diría Thomas Hobbes (2004: 131): "Donde no hay ley, no hay injusticia". En el estado de naturaleza no hay bueno ni malo, justo ni injusto. Pero cuando se forma el derecho, ya no se puede desinventar. Se le puede querer reemplazar por otras identidades formadas también operacionalmente, como cuando se presiona a los jueces políticamente, cuando se los amenaza con violencia o se los compra con dinero, pero en todos esos casos son otros los sistemas que intentan resolver los problemas propios que ellos mismos se inventaron en operaciones de enlace comunicativo recurrentes.

En un sentido más general, la operación es el punto ciego de la observación. El sistema opera y en la operación se construye la observación que permite indicar al sistema (Mascareño 2010). Es común la calificación de la teoría de Luhmann como una teoría constructivista. El propio Luhmann (2005b) la sostiene. Pero también es incompleto quedarse solo con 
ello, pues si bien la observación construye el mundo social y también el mundo psíquico, subyace a esta construcción (a las múltiples construcciones de mundo) la ultra-realidad de la operación. Esta entrega garantías de que la construcción producida no es un espectro o una aparición espontánea. La observación, de la conciencia o de la comunicación, acontece, es un evento en el mundo porque es producto de la operación. Esse sequitur operari. Por ello, también para Luhmann no es problemático distinguir entre la autonomía de los sistemas psíquicos y la autonomía de la sociedad. Mientras la segunda opera por medio de la comunicación, la primera lo hace por medio de la intencionalidad de la conciencia (Luhmann 2005c). Y ambas están acopladas a través de las variadas opciones del lenguaje y de las interrelaciones de vivencias y acciones entre alter y ego (Luhmann 2007). Como en Bourdieu, también en Luhmann, tanto las estructuras de la conciencia como las estructuras sociales que se forman en operaciones cumplen una función limitacional: proponen un horizonte de posibilidades para la generación operativa de elementos, pero no llegan a determinar la operación, como podría acontecer con la esencia real del homo sapiens.

De todos modos, siempre se corre el riesgo de interpretar a Luhmann por los resultados, con prescindencia del mecanismo operativo. Con esto quiero decir que se lo puede ver como el sociólogo de los sistemas funcionales estables que coercionan top-down a los individuos, sin atender a la operación que los produce -por ejemplo, Archer (2013). Cuando se lo ve así, no hay otra posibilidad que parezca estructuralismo. Sin embargo, puesto que la teoría se funda en la operatividad, los sistemas son altamente inestables y regulan su inestabilidad por medio de la inestabilidad y oscilación de la operación. Si es algo, la estabilidad solo podría ser una fórmula de contingencia sistémica, se aspira a ella, pero se sabe que no se puede alcanzar: las finanzas producen los derivados para estabilizar sus riesgos, pero los derivados producen más riesgo; el arte exige autenticidad para validarse como novedad, pero la autenticidad lleva constantemente a observar el arte producido y a la intertextualidad; la política occidental debe mostrar credenciales democráticas para ser aceptada, pero en nombre de eso muchas veces se violan derechos democráticos o se formulan democracias con múltiples apellidos: burguesa, liberal, popular, plebiscitaria, protegida, participativa, discursiva, agonista, representativa. Una 'inflación democrática' que termina por inestabilizar su valor.

Un segundo riesgo, que me parece más serio que el anterior, es entender la autopoiesis sistémica como una red de contenido perfecto (Spen- 
cer-Brown 1979) que inmuniza a los sistemas frente a la contingencia de ellos mismos y de su entorno. Si las cosas se entienden de este modo, entonces el sistema se reifica y sus estructuras actuales pasan a ser estructuras necesarias que deben ser mantenidas a toda costa. Jürgen Habermas (1971) hizo popular esta forma de comprensión de la teoría de Luhmann. En ella, el contenido del sistema (Bestand) es anterior a la operación. Lo que esta interpretación no observa es que la operación de autopoiesis (comunicación en sistemas sociales, intencionalidad en sistemas psíquicos) busca permanentemente suplir la incompletitud estructural y contingencia del sistema por medio de la simulación de contenidos perfectos y estructuras estables (Stäheli 2000; Mascareño 2016). El observador que ve un sistema en equilibrio es víctima de un engaño sistémico, de una demostración de la fuerza semántica del sistema que oculta su indeterminación última y que solo puede hacerse visible 'para todos' cuando el sistema comienza a reiterar irreflexivamente su propio éxito por medio de redundancias que, luego de un determinado punto, ya no es capaz de procesar. Estos son los momentos de crisis en sistemas sociales complejos (Mascareño et al. 2016). Tales momentos muestran la incompletitud de las estructuras y, a la vez, hacen evidente que es la propia operación sistémica la que lleva esas estructuras hasta el extremo de destruirlas en nombre de la continuidad de la operación, como en el caso de las crisis financieras o las revoluciones políticas, en las que solo cambian los criterios de limitacionalidad, pero la operación permanece.

\section{CONCLUSIÓN}

Las comparaciones teóricas pueden tener distintos fines. Uno puede enfocarse en lo que diversas teorías dicen acerca de un determinado ámbito de la realidad, puede observar el tratamiento que dan a un concepto que parece transversal, puede reconstruir el diagnóstico de época que tales teorías dan al mundo que experimentan o puede también preguntarse por las bases filosóficas y epistemológicas que subyacen a sus distinciones. En esta comparación he seguido una alternativa distinta: no he observado sus resultados ni sus antecedentes, sino las distinciones que usan para explicar cómo emerge genéticamente lo social. Esto es lo que indico con el concepto de operación. Por ello las llamo teorías operativas. Varias conclusiones pueden sacarse de esto. 
Primero, las teorías operativas son teorías acerca de la génesis de lo social. Ofrecen una explicación del mecanismo que produce lo social sin presuponer formas a priori que adquieran el carácter de fuente última de lo social. Para ellas, la sociedad emerge en la operación del mecanismo. En sus arquitecturas teóricas privilegian conceptos como emergencia, contingencia, praxis, diferencia, indeterminación, antes que otros como trascendencia, necesidad, idealidad, unidad o determinación. Consideran a las estructuras como derivados de la operación y les otorgan una función de limitacionalidad para las formas que lo social adopte. En tanto identifican un mecanismo generativo, sus rendimientos parciales tienen que ajustarse a esa dinámica. Eso las hace totalizadoras en su capacidad de interpretación, por tanto, diversas en sus elementos y en las relaciones entre esos elementos, pero comparables en su forma y en algunos casos compatibles en aspectos generales de sus teorías parciales.

Segundo, si la operación consiste en mostrar cómo emerge el fenómeno social que se quiere explicar, entonces distinciones clásicas de las ciencias sociales, como es la distinción micro/macro, carecen de sentido para las teorías operativas. Esta distinción da a entender que las relaciones entre personas tendrían una lógica distinta a las relaciones entre estructuras o sistemas. Las teorías operativas hacen evidente (esa es precisamente su tarea) la indisociabilidad de tales dimensiones. Las luchas en los campos no son un simple resultado de las prácticas. Son prácticas. Estructura y agencia se pueden distinguir analíticamente; se les puede atribuir incluso poderes causales distintos, pero la agencia no es pequeña escala ni la estructura es gran escala. La operación las hace comportarse como unidad de la diferencia. Los sistemas sociales simplemente dejan de funcionar sin la contribución de los sistemas psíquicos a la comunicación. Dicho en términos luhmannianos, en cada interacción está contenido el mundo, no como potencialidad, sino como reproducción del mundo en la interacción. Los metodólogos probablemente sean responsables de la distinción micro/macro, pero la teoría no puede dejarse guiar por ella -como sí lo hizo Robert K. Merton con sus 'teorías de alcance medio' que habrían servido para conectar ambas dimensiones. Las teorías operativas no se representan el mundo bajo este esquema, porque en la operación existe solo un mundo, que en todo caso puede aceptar distintas versiones de cómo llega a ser mundo.

Tercero, el énfasis en la operación, el mecanismo generativo y la emergencia hacen que el tiempo cumpla un rol central para las teorías operativas. No obstante, tampoco el tiempo puede ser entendido aquí como 
una categoría a priori; por el contrario, este emerge en la operación. El tiempo es inmanente a la relacionabilidad de la praxis, a la lucha en los campos, a los ciclos morfogenéticos e incluso a los morfoestáticos, a la comunicación como constelación de tres selecciones, a la intencionalidad noética de la conciencia. No se toma en préstamo desde un exterior ni se comprende como un plano pre-dado sobre el cual sostener la operación. La operación constituye el tiempo; la operación 'es' el tiempo. Esto permite sacar a la luz múltiples paradojas temporales de la modernidad -la contemporaneidad de lo no contemporáneo, la aceleración y sus islas de desaceleración, la inalcanzabilidad del futuro, la inaprehensibilidad del presente, la sincronía de capas históricas- sin tener que ocultarlas tras las poderosas fórmulas semánticas modernas del progreso, la modernización y el desarrollo, que unifican la experiencia del tiempo siempre en beneficio de quienes se sitúan arriba y adelante.

Cuarto, para las teorías operativas tampoco el espacio es un a priori. La dimensión material del mundo también es apropiada operacionalmente por la sociedad en forma de límites políticos, jurídicos y militares, y también puede ser operacionalmente desmaterializada en redes translocales, transnacionales o supranacionales en diversos ámbitos de interacción, organizaciones o sistemas sociales. Para las teorías operativas, el espacio cuenta más bien como un vector de relaciones, como un 'espacio de fase' que se constituye en la operación y se modifica, emerge o se disuelve con ella y por ella.

Quinto, si la operación muestra la génesis de lo social, entonces las teorías operativas no pueden ser inmanentemente normativas. Las teorías normativas definen un parámetro de clasificación del tipo conformidad/desviación que actúa como fuente de lo que puede ser social y de lo que se desvía de la norma social. Pero ello deja sin explicar qué es lo que origina la norma. Para resolver ese problema hay que suponer que las normas son previas a la operación, lo que no es aceptable para teorías operativas. La religión formuló teorías de este tipo, luego lo hizo el derecho natural y en el siglo XX fue el turno del estructuralismo. Puesto que las teorías operativas son genéticas, ellas dan cuenta de la emergencia social de Dios, de lo que en cada época se denomina naturaleza humana y de las estructuras sociales que reclaman universalidad.

Sexto, nada impide, sin embargo, que las teorías operativas puedan, por supuesto, reconocer las normas que la sociedad produce y que incluso puedan hacer formulaciones normativas. Bourdieu lo hace, en tanto su teo- 
ría sugiere que el problema fundamental de la modernidad es la distribución (de capitales) y la participación (en campos); Archer lo hace en términos de la realización de planes de vida de las personas, y Luhmann en forma de desenmascaramiento de paradojas sistémicas que ocultan a la propia sociedad que las cosas pueden ser de otro modo. La ventaja con las teorías operativas es que sabemos de dónde viene la norma y sabemos que, porque viene de una operación, su trasgresión es una 'desviación normal'. Esto incluso nos puede dar más motivación para mantener la norma, o también para transgredirla cuando lo consideramos apropiado.M

\section{REFERENCIAS}

Aguilar, O. (2014). Agencia y estructura en la teoría del habitus de Pierre Bourdien. Tesis para la obtención del título de PhD, Universidad Alberto Hurtado, Santiago de Chile.

Alladi, S. (2013). The Reflexive Turn: The Rise of First-Person Ethnography. The Sociological Quarterly, 54(1), 3-8.

Archer, M. (2004). Being Human. Cambridge: Cambridge University Press.

Archer, M. (2007). Making Our Way Through the World. Cambridge: Cambridge University Press.

Archer, M. (2009). Teoría social realista: el enfoque morfogenético. Santiago de Chile: Ediciones Universidad Alberto Hurtado.

Archer, M. (2012). The Reflexive Imperative in Late Modernity. Cambridge: Cambridge University Press.

Archer, M. (2013). Social Morphogenesis and the Prospects of Morphogenic Society. In M. Archer (Ed.), Social Morphogensis (pp. 1-22). Dordrecht: Springer.

Archer, M. (2015). The Relational Subject and the Person: Self, Agent, and Actor. In P. Donati \& M. Archer, The Relational Subject (pp. 85-122). Cambridge: Cambridge University Press.

Bhaskar, R. (1989). Re-claiming Reality. London: Verso.

Bourdieu, P. (1997). Razones prácticas. Barcelona: Anagrama.

Bourdieu, P. (2002). Campo de poder, campo intelectual. Itinerario de un concepto. Buenos Aires: Montressor.

Bourdieu, P. (2007). El sentido práctico. Buenos Aires: Siglo XXI.

Bourdieu, P. \& Wacquant, L. (1992). An Invitation to Reflexive Sociology. Cambridge: Polity Press.

Cadenas, H. (2012). El sistema de la estructura. Estructuralismo y teoría de sistemas. Cinta de Moebio, 45, 204-214.

Derrida, J. (1997). Of Grammatology. Maryland: The Johns Hopkins University Press.

Donati, P. (2011). Relational Sociology. London: Routledge.

Emirbayer, M. (1997). Manifesto for a Relational Sociology. The American Journal of Sociology, $103(2), 281-317$.

Habermas, J. (1971). Theorie der Gesellschaft oder Sozialtechnologie? Eine Auseinandersetzung mit Niklas Luhmann. In J. Habermas \& N. Luhmann, Theorie der Gesellschaft oder Sozialtechnologie? (pp. 142-290). Frankfurt aM: Suhrkamp.

Hobbes, T. (2004). Leviatán. Buenos Aires: Losada

Luhmann, N. (1984). Soziale Systeme. Frankfurt aM: Suhrkamp. 
Esse sequitur operari, o el nuevo giro de la teoría sociológica contemporánea: Bourdieu, Archer, Luhmann $\mid 73$

Luhmann, N. (1998). Los medios generalizados y el problema de la doble contingencia. In N. Luhmann, Teoría de los sistemas sociales (articulos) (pp. 9-73). México DF: Universidad Iberoamericana.

Luhmann, N. (2005a). Identität -was oder wie?. In N. Luhmann, Soziologische Aufklärung 5 (pp. 15-30). Wiesbaden: VS Verlag.

Luhmann, N. (2005b). Das Erkenntnisprogramm des Konstruktivismus und die unbekannt bleibende Realität. In N. Luhmann, Soziologie Aufklärung 5 (pp. 31-57). Wiesbaden: VS Verlag.

Luhmann, N. (2005c). Die operative Geschlossenheit psychischer und sozialer Systeme. In N. Luhmann, Soziologische Aufklärung 6 (pp. 26-37). Wiesbaden: VS Verlag.

Luhmann, N. (2007). La sociedad de la sociedad. México DF: Herder.

Luhmann, N. (2013). La moral de la sociedad. Madrid: Trotta.

Marttila, T. (2015). Post-Foundational Discourse Analysis. From Political Difference to Empirical Research. London: Palgrave.

Marx, K. (1978). Theses über Feuerbach. In Karl Marx und Friedrich Engels Werke (pp. 5-7). Berlin: Dietz Verlag.

Mascareño, A. (2008). Acción, estructura y emergencia en la teoría sociológica. Revista de Sociología, 22, 217-256.

Mascareño, A. (2010). Construct this! O por qué el constructivismo sistémico es real. Revista $M A D, 23,9-24$.

Mascareño, A. (2016). La incompletitud de la autopoiesis: Irritación, codificación y crisis. Metapolitica, 20(92), 26-35.

Mascareño, A., Goles, E., \& Ruz, G. (2016). Crisis in Complex Social Systems. Complexity 21(2), 13-23. DOI 10.1002/cplx.21778.

Marquard, O. (2003). Entlastungen. Theodizeemotive in der neuzeitlichen Philosophie. In O. Marquard, Zukunft braucht Herkunft. Philosophische Essays (pp. 124-145). Stuttgart: Reclam.

Maturana, H. (2009). La realidad: ¿Objetiva o construida? Barcelona: Anthropos.

Nassehi, A. (2004). Sozialer Sinn. In A. Nassehi \& G. Nollmann (Eds.), Bourdieu und Lubmann. Ein Theorievergleich (pp. 155-188). Frankfurt aM: Surhkamp.

Nassehi, A. (2006). Der soziologische Diskurs der Moderne. Frankfurt aM: Suhrkamp.

Parsons, T. (1968). La estructura de la acción social. Madrid: Guadarrama.

Parsons, T. (1991). The Social System. London: Routledge.

Parsons, T., Bales, F., \& Shils, E. (1970). Apuntes sobre la teoría de la acción. Buenos Aires: Amorrortu.

Rescher, N. (1996). The Promise of Process Philosophy. Process Studies, 25, 55-71.

Spencer-Brown, G. (1979). Laws of Form. New York: E.P. Dutton.

Stäheli, U. (2000). Sinnzusamenbrüche. Eine dekonstruktive Lektüre von Niklas Lubmanns Systemtheorie. Weilerwist: Velebrück Wissenschaft.

Varela, F., Thompson, E., \& Rosch, E. (1993). The Embodied Mind. Cambridge, Mass.: The MIT Press.

Wacquant, L. (1992). Toward a Social Praxeology: The Structure and Logic of Bourdieu's Sociology. In P. Bourdieu \& L. Wacquant, An Invitation to Reflexive Sociology (pp. 1-60). Cambridge: Polity Press.

Weber, M. (1992). Economía y sociedad. México DF: Fondo de Cultura Económica.

White, H. (2008). Identity and Control. How Social Formations Emerge. Princeton: Princeton University Press.

Wittgenstein, L. (1986). Philosophical Investigations. Oxford: Basil Blackwell. 


\section{SOBRE EL AUTOR}

Aldo Mascareño. PhD en Sociología, Universidad de Bielefeld, Alemania. Profesor Titular de la Escuela de Gobierno de la Universidad Adolfo Ibáñez, Santiago de Chile y Director del Centro de Investigación Núcleo Milenio Modelos de Crisis (NS 130017).

\section{CONTACTO}

aldo.mascareno@uai.cl

Recibido: enero 2017

Aceptado: junio 2017

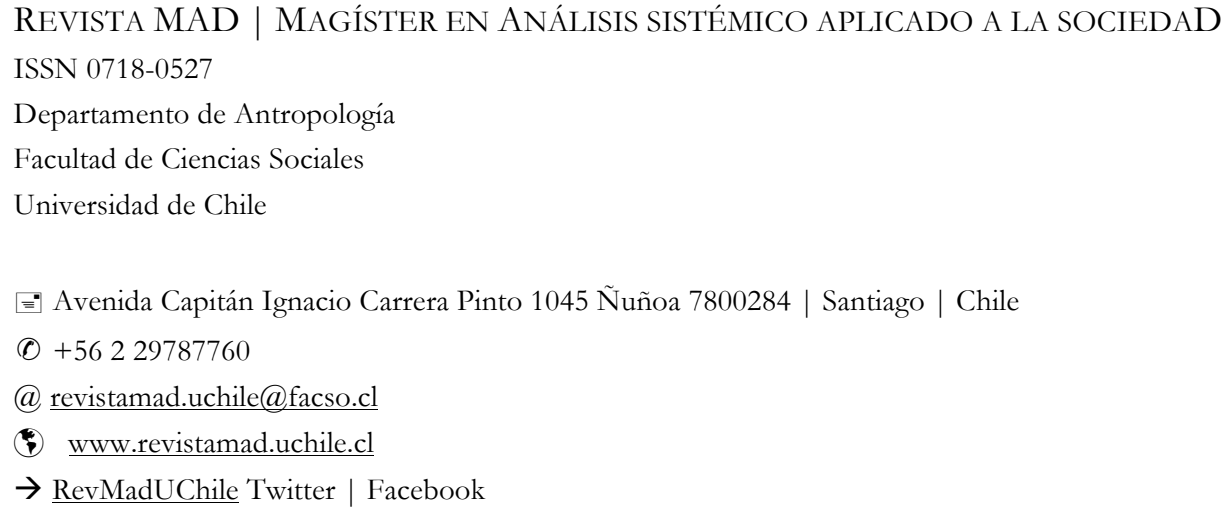

